\title{
Radiation protection study for a reloading operation of cobalt 60 sources in the Moroccan ionisation station using the GEANT4 Monte-Carlo simulation code
}

\author{
Adil Aknouch ${ }^{1, *}$, Youssef El-ouardi ${ }^{2}$, Mohammed Mouhib $^{3}$, Rajaa Sebihi $^{4}$, Abdelmajid Choukri ${ }^{1}$ \\ ${ }^{1}$ Department of Physics, Nuclear Physics and Techniques Team, Faculty of Science, Ibn Tofail University, Kenitra, Morocco \\ ${ }^{2}$ Department of Physics, Faculty of Sciences Dhar El-Mahraz, Sidi Mohamed Ben Abdellah University, Fez, Morocco \\ ${ }^{3}$ National Institute for Agronomical Research (NIAR), Irradiation Facility of Boukhalef (SIBO), Tangier, Morocco \\ ${ }^{4}$ Department of Physics, Faculty of Sciences, Mohammed V University, Rabat, Morocco
}

\begin{abstract}
The operation of reloading the irradiators is considered among the tasks requiring high radiation protection monitoring, to protect the intervening manipulators, the public and the environment. Morocco is among the countries that have a cobalt irradiator, installed at the National Institute of Agricultural Research (NIAR) of Tangier, to carry out research in the field of agronomy. In the beginning, the irradiator used low doses of activity for the study of products only, for treatment of high doses. The NIAR carried out a reload to increase the activity. To perform this, a temporary pool was installed inside the irradiation room to handle the sources safely. A radiation protection study is necessary to ensure the safe operation. This operation requires a height level of exposure. To ovoid the exposer risk, it is proposed to use the Monte Carlo method thanks to its reliability in the dosimetric calculation. This article presents a radiation protection study of the Moroccan irradiator reloading operation using the GEANT4 Monte-Carlo Simulation Code.
\end{abstract}

\section{Introduction}

NIAR of Tangier has a panoramic cobalt-60 irradiator to carry out research in the fields of food conservation, protection and improvement of plants by induction of mutations.

At the beginning, the irradiator contains 2 radioactive sources of sufficient activity only for the study of products which require low doses, but to carry out the treatments which require height doses, it takes a lot of irradiation time to reach the desired dose, and that will influence the quality of the products. To solve this problem, the NIAR decided to reloading the irradiator by adding 6 other sources to reach an activity of $60000 \mathrm{Ci}$ on the day of reloading.

The reloading operation was carried out in a water pool temporarily installed in the irradiation cell, which will allow the transfer of the source pencils from the supplier's transport container to the SV68 irradiator container at the NIAR site. So high radiation protection monitoring for this operation is mandatory, but it takes a lot of means and the risk of exposure is more likely. To solve these problems and strengthen the radiation protection of this operation, the Monte Carlo simulation was used. Thanks to its reliability and efficiency in dosimetric calculations [1], the Monte-Carlo simulation succeeded in solving many dosimetry and radiation protection problems for ionization stations around the world [2-5]. The Moroccan ionization station also takes advantage of this method to control the dose distribution in the irradiation cell, these studies carried out by Aknouch [6] and Elouardi [7]. Another Monte Carlo study was carried out by Aknouch [8] to improve the irradiation technique by predicting the packaging of the products in order to obtain a better dose homogeneity.

The objective of this article is to use the GEANT4 Monte-Carlo simulation code to predict the distribution of equivalent dose rates in the temporary pool and in the irradiation cell during the reloading operation in order to reinforce radiation protection.

\section{Materials and methods}

\subsection{Description of the irradiation cell and the temporary pool}

The ionization cell is the part in which the ionization of the products takes place, it is parallelepiped in shape 5.8 $\mathrm{m}$ wide, $6.1 \mathrm{~m}$ long, and 2.6 meters high. The walls of the cell with a thickness of $1.63 \mathrm{~m}$ allow the biological protection of operators and the environment from the effects of gamma radiation.

The water pool will be placed in the cell through the hole in the ceiling cap. The size of the pool will match the size of the hole correctly. The external dimension of the

*Corresponding author: adil.aknouch@uit.ac.ma 
pool is $1.28 \mathrm{~m}$ while the diameter of the cavity is $1.3 \mathrm{~m}$, the tolerance of $20 \mathrm{~mm}$ will allow a comfortable and safe operation with the pool. Since the technical room does not allow the installation of the pool in one room. So, the pool is prepared in two parts, and the latter are unified with the welding on site. The thickness of the stainless-steel wall of the pool is $4 \mathrm{~mm}$, which will allow a simple and safe welding procedure.

\subsection{Description of the reloading operation}

We can summarize the technique of reloading in the Moroccan irradiator in the following steps:

- Removal of the SV68 container installed at the irradiation cell, then a temporary swimming pool will be installed inside the irradiation cell and filled with water.

- A transport container which contains three source pencils is introduced into the pool. The upper cover of the transport container will be removed and the 3 new source pencils will be removed from the container cavity [Figure 1a]. with their own source cage provided by the supplier and will be placed in the cavity on a table. The same procedure will be carried over with the second transport container.

- As soon as the 6 new sources are loaded into the pool. The two old sources will be removed and placed in the table cavity [Figure $1 \mathrm{~b}$ ]. Then, the SV-68 container will be temporarily removed from the pool, to allow sufficient clearance for the safe source loading operation. When all the sources are gathered on the table, a second table is installed and the source holder of the SV- 68 container will be placed on the table system. This allows easy and safe source loading underwater [Figure 1c].

-When all the sources are installed in the source holder of the SV-68 irradiator and the support is fixed to the upper plug of the SV-68, the assembly will be placed in the cavity of table I [Figure 1d]. Then Table II will be removed from the pool and the SV-68 container will be returned to the pool. Then, the cap and source holder will be returned to the SV-68 container [Figure 1e].

- After the completion of the reloading operation, the SV68 container will be removed from the pool [Figure 1f], The water will be pumped from the pool, then, the pool will be cut in two pieces and removed from the cavity of the bunker.

- The SV-68 container will be placed in its normal position and reinstall the other equipment.

\subsection{Modeling of the reloading operation using the Geant4 code}

To carry out the modeling of the recharging operation, we used the code GEANT4 version 10.5 (December 7, 2018), in order to calculate the equivalent dose rates distribution as a function of the depth in the provisional pool and establish a map dosimetric inside the irradiation cell during recharging when all sources are placed on the table.

GEANT4 (GEometry ANd Tracking 4) is one of the most widespread and used simulation codes, because it is very powerful and flexible for its users, this code developed at CERN and based on Monte-Carlo objectoriented for particles transport of through matter [9]. Being object oriented, it uses the notion of classes and objects. The user derives from classes to describe the primary particle generator, the geometry and choose the physical process. The latter included the photoelectric effect, Compton and Rayleigh scattering, pair production, positron annihilation, ionization, Bremsstrahlung, synchrotron and transition radiation, Auger electrons, absorption, scintillation and fluorescence, refraction, reflection and Cherenkov effect [10-11].

In this work, geometry and materials similar to the experimental situation, to achieve precision as much as possible. To calculate the equivalent dose rate by the GEANT4 code, we used the relation (1):

$$
\dot{H}_{T}=w_{T, R} \times \dot{D}_{T, R}=w_{T, R} \times \frac{2 \times E \times A \times C \times t}{N \times m}
$$

With:

$\mathrm{W}_{\mathrm{T}, \mathrm{R}}$ : Radiation weighting factor(Sv/min)

$\dot{D}_{T, R}$ : Dose rate absorbed averaged over the tissue, due to the incident radiation $\mathrm{R}$ ( $\mathrm{Gy} / \mathrm{min})$

E: Total energy deposited in a dosimeter $(\mathrm{MeV})$,

$\mathrm{C}$ : Conversion factor $(\mathrm{J} / \mathrm{MeV})$, equal to $1.602 \times 10^{-10}$,

A: Activity in (Bq),

$\mathrm{N}$ : Number of generated events, equal to $2.10^{9}$,

2: Factor to take into account two emitted photons by the 60Co source,

t: The irradiation time, equal to $60 \mathrm{~min}$.

\section{Results and discussion}

To reinforce the radiation protection of the recharging operation, by determining the optimal depth for handling the sources safely in the temporary pool, and showing the risk of being inside the irradiation cell, a calculation of the distribution of equivalent dose rates in the latter and in the swimming pool was carried out using the GEANT4 code. The figure 3 shows the distribution of equivalent dose rates in the temporary pool when all the sources are placed on the tables. This will make it possible to estimate the equivalent dose rate on the surface of the temporary pool where the manipulators are located, and therefore to determine the adequate depth of water to manipulate the sources, this study will also be used to optimize the volume from the pool.

Figure 4 shows the distribution of equivalent dose rates inside the irradiation cell when handling sources. Therefore, the latter shows that during handling the sources in the temporary pool the equivalent dose rates in the cell are very high at the level of radiation protection, which shows that the risk of being inside the cell during the reload operation is very high.

This study gives the optimal depth for handling sources safely, and shows the risk of ending up inside the irradiation cell during reloading. 


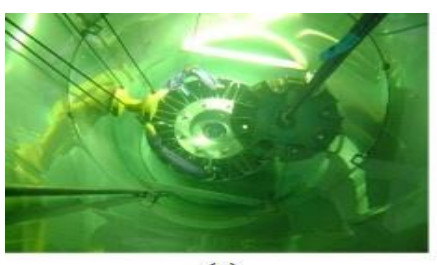

(a)

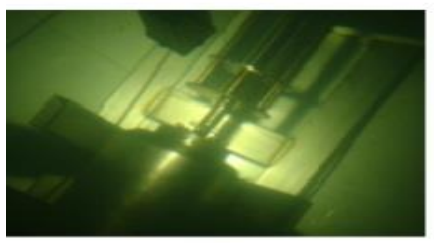

(d)

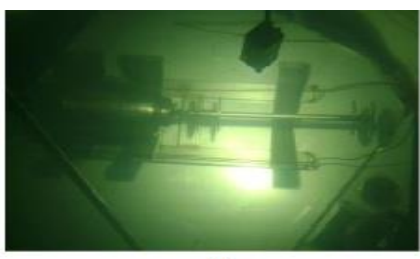

(b)

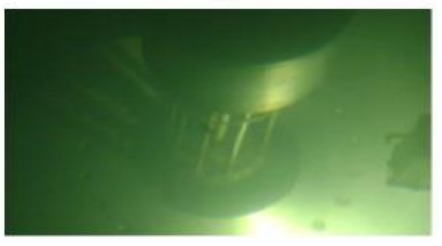

(e)

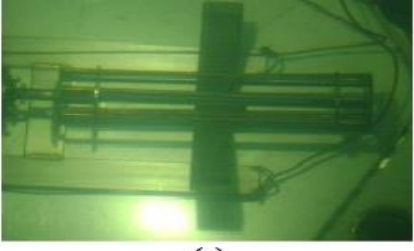

(c)

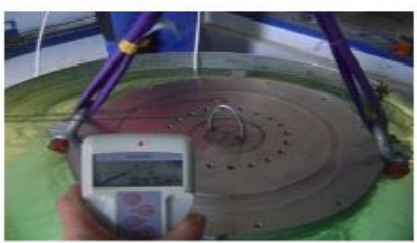

(f)

Fig.1. The steps of the loading operation carried out at NIAR

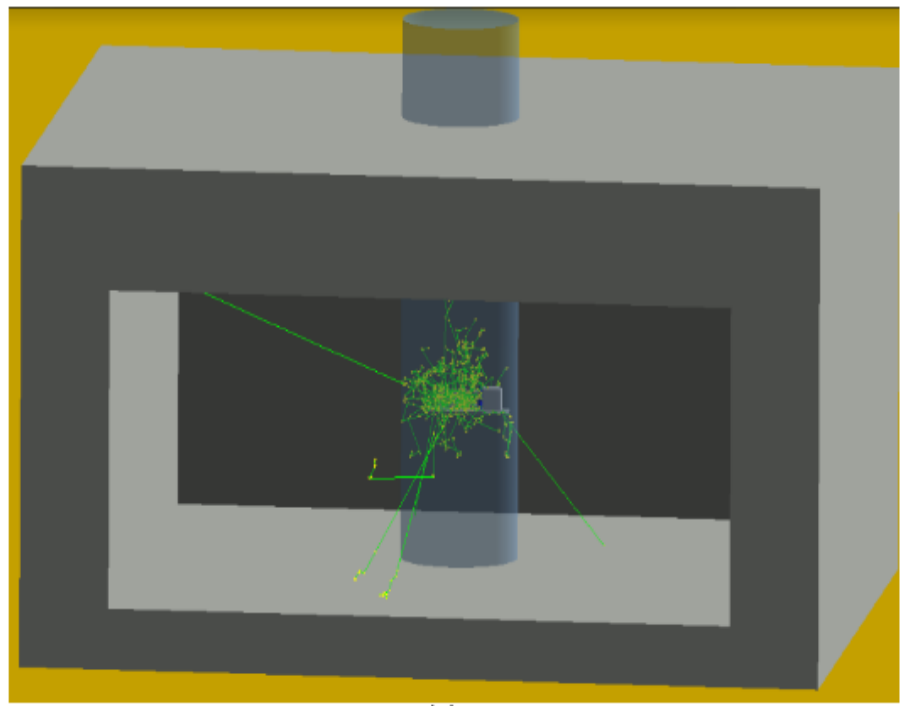

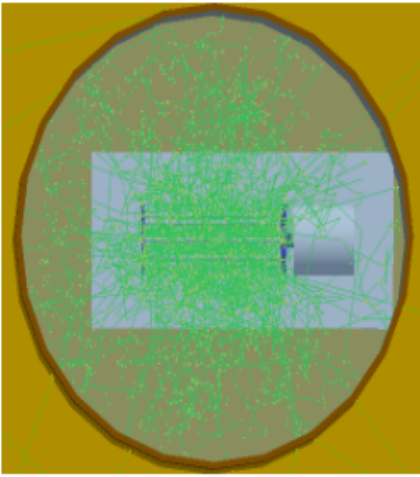

(b)

(a)

Fig.2. (a) 3D view of the irradiation cell GEANT4 model. (b) . the eight sources in the pool. Plane XY

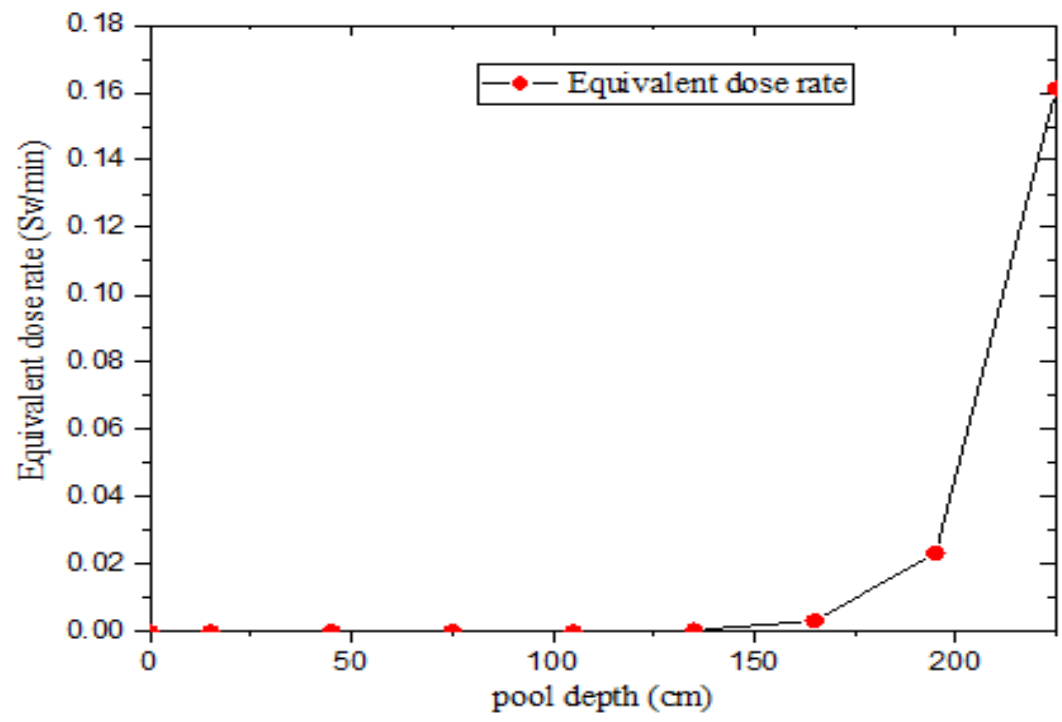

Fig.3. distribution of equivalent dose rates as a function of depth in the temporary pool 


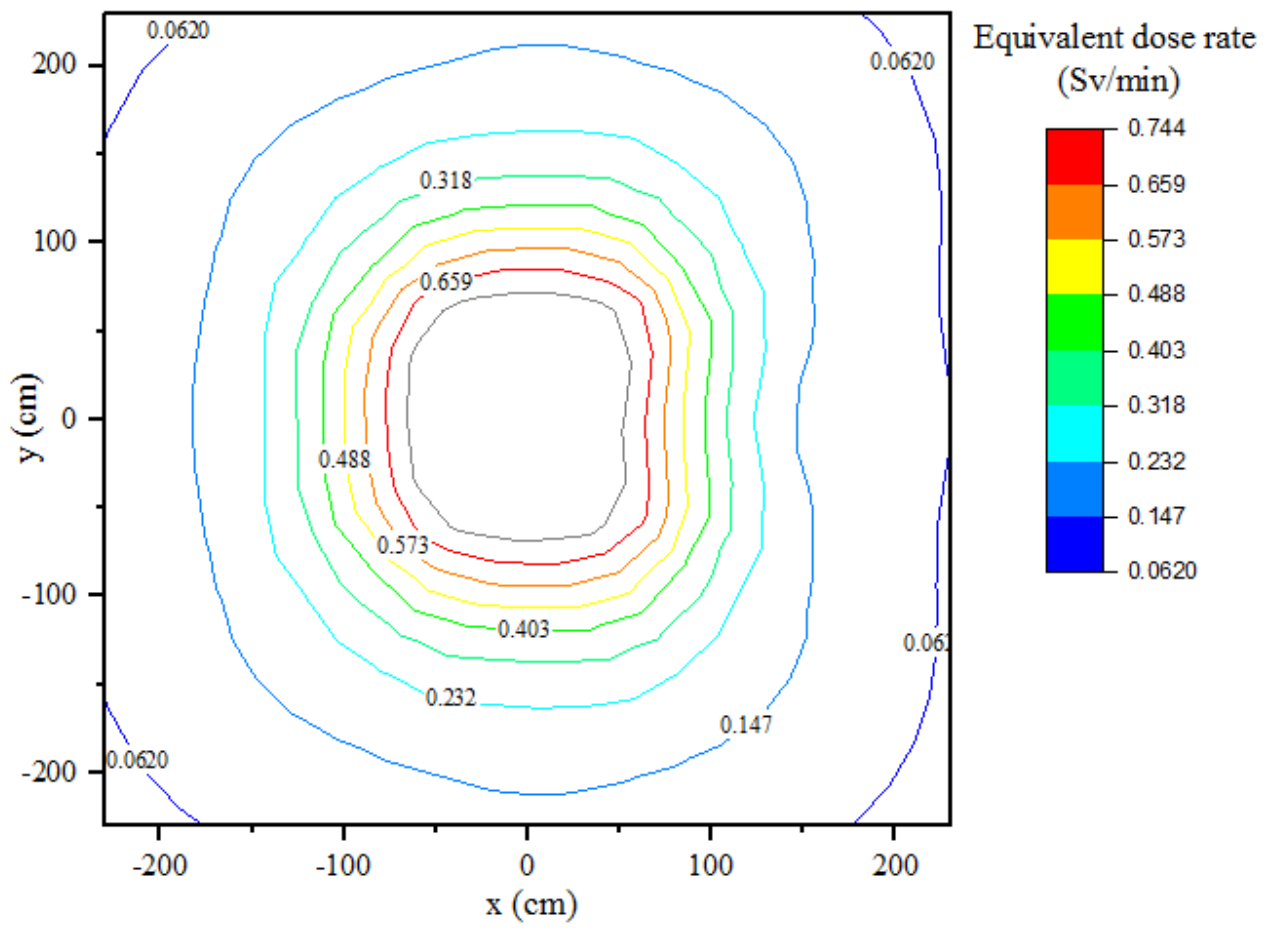

Fig.4. Distribution of equivalent dose rates in the irradiation cell during the reloading operation

\section{Conclusion}

This article is another advantage of the Monte Carlo simulation for research irradiators, which is the reinforcement of the radiation protection of the operations of reloading of the sources, by preventing the adequate depth to handle the sources in full safety, and to determine the places where the risk exposure is very high.

\section{References}

1. M. Lydia, «Dosimétrie personnalisée par simulation Monte Carlo GATE sur grille de calcul. Application à la curiethérapie oculaire. ", thèse, Université Blaise Pascal - Clermont-Ferrand II(2005).

2. K. Khattab, M. Boush, et H. Alkassiri, Ann. Nucl. Energy, vol. 58, p. 110-112(2013)

3. Y. H. Kim et J. W. Park, J. Nucl. Sci. Technol., vol. 45, p. 325-328, (2008)

4. M. Rodriguez Gual, A. Z. Mesquita, E. Ribeiro, et P. A. Grossi, Sci. Technol. Nucl. Install., vol. 2017(2017)

5. T. Van Hung et T. Khac An, Appl. Radiat. Isot., vol. 68, p. 1104-1107(2010)

6. A. Aknouch, M. Mouhib, R. Sebihi, A. Didi, Y. Elouardi, A. Boubekraoui et A, Choukri, Mosc. Univ. Phys. Bull, vol. 75, No. 1, pp. 35-38 (2020)

7. Y. El-ouardi, A. Dadouch, A. Aknouch, M. Mouhib, A. Didi, Mosc. Univ. Phys. Bull, (to be published)

8. A. Aknouch, Y. El-ouardi, M. Mouhib, R. Sebihi, A. Didi, et A, Choukri, Mosc. Univ. Phys. Bull, (to be published)
9. E. Salama et A. Maher, J. Phys. Conf. Ser., vol. 1253, p. 012032(2019)

10. «Introduction to Geant4 », p. 19.

11. V. Belousov, A. A. Kalachev, G. A. Krusanov, et A. P. Chernyaev, Mosc. Univ. Phys. Bull., vol. 70, p. 416-422(2015) 